\title{
Illuminance $\left(\mathrm{Im} \mathrm{M}^{-2}\right)$ of Compact 20W-HCFL Tube
}

\section{Lyuji Ozawa}

The Japanese Government licensed consultant in science, Beijing, China

\author{
Email address: \\ rotsun4@hotmail.com
}

\section{To cite this article:}

Lyuji Ozawa. Illuminance ( $\left(\mathrm{m} \mathrm{M}^{-2}\right.$ ) of Compact 20W-HCFL Tube. Science Research. Vol. 3, No. 4, 2015, pp. 170-179.

doi: 10.11648/j.sr.20150304.14

\begin{abstract}
It has found that the study on the FL tubes has made with the deliberately modified Ulbricht Spheres for the determination of the luminous efficiency $(\mathrm{lm} \mathrm{W})$ that uses in the study on the colorimetry and that invalidates the study on the light sources. The study on the light sources should make with either illuminance $\left(1 \mathrm{~m} \mathrm{~m}^{-2}\right)$, or luminance $\left(\mathrm{cd} \mathrm{m}^{-2}\right)$ and irradiance $\left(\mathrm{W} \mathrm{m}^{-2}\right)$. The rooms in the house should be illuminated with the daytime scenery with the slightly overcastting sky $\left(330 \mathrm{~lm} \mathrm{~m} \mathrm{~m}^{-2}\right)$ that human eyes adjust for 5 million years. The illuminance $\left(\mathrm{lm} \mathrm{m}^{-2}\right)$ of the commercial compact-20W-HCFL tubes, which have selected from the stores, has determined with the corrected Ulbricht Sphere. The selection criterion is the temperature of the positive column above $60^{\circ} \mathrm{C}$ of the lighted FL tubes. The examined compact 20W-HCFL tubes have the illuminance $(5700 \pm 300$ $1 \mathrm{~m} \mathrm{~m}^{-2}$ ). Two compact 20W-HCFL tubes that set in a large opaque plastic cover comfortably illuminate the $30 \mathrm{~m}^{2}$ with the $380 \mathrm{~lm}$ $\mathrm{m}^{-2}$. The determined $\mathrm{W}$ does not involve in the generation energy of the lights in the FL tubes. The reduction of the $\mathrm{W}_{\text {act }}$ of the external AC driving circuits remains for a future study.
\end{abstract}

Keywords: FL Tube, Illuminance, Light Source, Ulbricht Sphere, Luminous Efficiency

\section{Invalidated Luminous Efficiency (Im $\left.W^{-1}\right)$ for Study on FL Tubes}

Traditionally, the performance of the commercial FL tubes has evaluated with the luminous efficiency $\left(\mathrm{lm} \mathrm{W}^{-1}\right)$ since the invention on 1928 [1, 2, and 3]. The luminous efficiency is only used in the study on the colorimetry. The capability of the light sources such as FL tubes and LEDs do not evaluate with the invalided luminous efficiency. The Ulbricht Sphere (integrating sphere) has deliberately modified for the determination of the invalidated luminous efficiency $\left(\mathrm{lm} \mathrm{W}^{-1}\right)$ of the FL tubes. The control panel of the modified Ulbricht Sphere gives only the lumen $(\mathrm{lm})$ and AC power consumption (W) for the calculation of the luminous efficiency $\left(1 \mathrm{~m} \mathrm{~W}^{-1}\right)$. The erroneous evaluations of the FL tubes by the luminous efficiency $\left(\mathrm{lm} \mathrm{W}^{-1}\right)$ led to a conclusion that the productions of the FL tubes were the mature technologies on 1970s. The scientists and engineers of the FL tubes have concluded no room for the further improvement of the quality of the FL tubes. The study on the FL tubes had terminated on USA before 1980. This is a wrong conclusion by the use of the invalided luminous efficiency $\left(\mathrm{lm} \mathrm{W}^{-1}\right)$ that is determined by the incorrectly modified Ulbricht Sphere.

The control panel of the right Ulbricht Sphere does not give the lumen and W. The Ulbricht Sphere has designed as the determination of one of the followings:

(1) Illuminance $\left(\mathrm{lm} \mathrm{m}^{-2}\right)$,

(2) Luminance $\left(\mathrm{cd} \mathrm{m}^{-2}\right)$, or

(3) Irradiance $\left(\mathrm{W} \mathrm{m}^{-2}\right)$,

The determinations depend on the kind of the light sensors installed in the hole on the inner wall of the Ulbricht Sphere. We have studied, as an example, on the illuminance $\left(\mathrm{lm} \mathrm{m}^{-2}\right)$ of the commercial compact-HCFL tubes by the right Ulbricht Sphere. It has found that the technologies of the FL tubes are not the mature technology.

This report is the study on the correction of the modified Ulbricht Sphere as the first step. The corrected Ulbricht Sphere determinate the illuminance $\left(\mathrm{lm} \mathrm{m}^{-2}\right)$ of the lighted FL tubes. The determined illuminance $\left(1 \mathrm{~m} \mathrm{~m}^{-2}\right)$ has verified the illumination of the room with the selected compact-20W-HCFL tubes. The energy of the AC electric power consumption $\mathrm{W}_{\text {act }}$ of the $\mathrm{AC}$ driving circuit isolates from the generation of the lights in the lighted FL tubes. By the corrections of the evaluation of the FL tubes, the lighted FL tubes have the unrivaled advantages that have concealed by the wrong evaluation of the FL tubes. The study on the FL tubes belongs to the vacuum physics, rather than analogues with the solid-state physics. 


\section{Correction of Improperly Modified Ulbricht Sphere}

The inner wall of the Ulbricht Sphere is covered with the layer of the white fine particles of calcium sulfate, $\mathrm{CaSO}_{4}$ that have the optical reflectance at around $95 \%$, not $100 \%$. The reflectance of $\mathrm{CaSO}_{4}$ slightly changes with the time by the adsorption of moisture from the air. The periodical maintenance requires for the precise determination. The lights from a light source multiply reflect on the inner wall of the Ulbricht Sphere. The multireflections of the lights result in the uniform illuminance $\left(1 \mathrm{~m} \mathrm{~m}^{-2}\right)$ on the entire area of the inner wall of the Ulbricht Sphere. The light sensor sets in a hole in the inner wall of the Ulbricht Sphere.

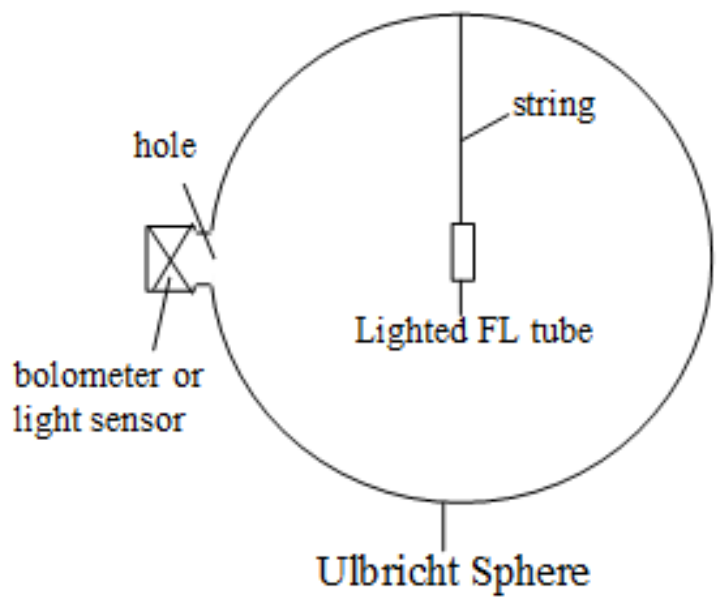

Figure 1. Illustration of fundamentals of arrangement of lighted FL tube and light sensor in hole at Ulbricht Sphere.

Figure 1 illustrates the fundamentals of the arrangement of the FL lamp and a hole for the light sensor. The hole size is usually $1 \mathrm{~cm}^{2}\left(=1 \times 10^{-4} \mathrm{~m}^{2}\right)$ that is given by CGS unit. The surface area of the inner wall of the Ulbricht Sphere is given by $4 \pi(d / 2)^{2}=\pi d^{2}$, where $d$ is inner diameter of the Ulbricht Sphere. In general, $\mathrm{d}$ of the Ulbricht Sphere is $2 \mathrm{~m}$. A basic study on the light source is made by the irradiance $\left(\mathrm{W} \mathrm{cm}^{-2}\right)$ where $\mathrm{W}$ is the light energy that is determined by the bolometer in the hole. The bolometer determines the energy of the lights from the light source. The irradiance $\left(\mathrm{W} \mathrm{m}^{-2}\right)$ in MKS unit is given by the multiplication of $1 \times 10^{4}$ to the detected irradiance ( $\mathrm{W} \mathrm{cm}^{-2}$ ) in CGS unit. The scientists and engineers, who are studying the FL tubes, take the $\mathrm{W}$ as the active power consumption $\mathrm{W}_{\text {act }}$ of the $\mathrm{AC}$ driving circuit. We take the $\mathrm{W}_{\text {act }}$ for the $\mathrm{AC}$ power consumption of the $\mathrm{AC}$ driving circuit. The $\mathrm{W}$ is the energy of the lights from the lighted FL tube for an avoidance of the confusion in this report.

In the practices of the FL tubes as the light sources, the lights are detected by the light sensors that adjust to the eye's sensibility (e.g., luminous curve) of the lights in the spectral wavelengths. The adjustment of the light sensor to the eyes' sensibility is made by the combinations of the light sensor and layers of the different optical filters. The original light sensor was the photomultiplier tube that has a high sensibility to the light detection. Someone makes a small correction for a removal of the direct light into the hole from lighted FL tube. The removal of the direct light from the light source had been made by setting a thin and small white-reflection plate in front of the light source. The reflection plate is slightly wider than the size of the light source. Figure 2 illustrates the reflection plate for the removal of the direct light from the lighted FL tube to the hole of the Ulbricht Sphere. However, the installation of the small reflection plate is unnecessary in the practice. The reason is the ratio of the hole size $\left(1 \times 10^{-4} \mathrm{~m}^{2}\right)$ to the total inner surface area $\left\{\right.$ e.g., $12.4 \mathrm{~m}^{2}\left(=4 \pi \mathrm{r}^{2}=\pi \mathrm{d}^{2}=3.1 \mathrm{x}\right.$ $\left.\left.2^{2} \mathrm{~m}^{2}\right)\right\}$ of the Ulbricht Sphere. The diameter of many of the practical Ulbricht Sphere for study on the FL tubes is $2 \mathrm{~m}$. The ratio of the hole size to the entire inner surface area of the Ulbricht Sphere is $8 \times 10^{-5}\left\{=1 \times 10^{-4} \mathrm{~m}^{2} \times\left(12.4 \mathrm{~m}^{2}\right)^{-1}\right\}$ that is negligible small for the consideration of the direct radiation into the hole in the Ulbricht Sphere. The variation of the illuminance of the produced FL tubes fall in a few $\%$, not $10^{-5}$. The installation of any reflection plate in the Ulbricht Sphere is unnecessary for the determination of the illuminance ( $\mathrm{lm}$ $\mathrm{m}^{-2}$ ) and luminance $\left(\mathrm{cd} \mathrm{m}^{-2}\right)$ of the study on the lighted FL tubes.

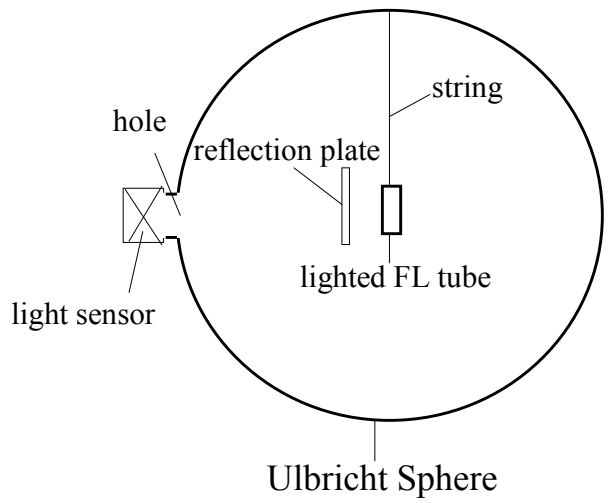

Figure 2. Reflection white plate in small size sets in front of lighted FL tube for avoidance of direct lights of FL tube into sensor hole.

The light sensors at present time are the solid light sensors that have the very low sensitivity as compared with that of the photomultiplier tube. It has guessed that the sensitivity of the solid light sensor in the early time has a very weak as compared with the sensitivity of the photomultiplier tube. Then, it might find a solution to set the combinations of a large reflection plate in front of the hole and an optical lens at the hole.

The large reflection plate disturbs the multi-reflection on the inner wall of the Ulbricht Sphere. Furthermore, the lens on the hole selectively collects the reflected lights on the large reflection plate. Consequently, the sensor in the hole detects the enhanced lights from the wide area of the reflected plate, as compared with the uniform light intensity on unit area (1 $\mathrm{cm}^{2}$ ) on the inner wall of the Ulbricht Sphere. Figure 3 illustrates the incorrect position and size of the reflection on the white plate that sets in front of the hole for the light sensor. The hole of the light sensor mainly receives the reflected lights from the large reflection plate, instead of the multiply reflected lights from the inner wall of the Ulbricht Sphere. The 
large plate in front of the hole blocks the reflected light from the inner wall of the Ulbricht Sphere. Consequently, the light sensor mainly detects the reflected lights on the large reflection plate and detects less multi-reflected lights on the inner wall of the Ulbricht Sphere.

This is a wrong arrangement for the determination of the illuminance $\left(1 \mathrm{~m} \mathrm{~cm}^{-2}\right)$ from the Ulbricht Sphere. The detected luminance from the Ulbricht Sphere does not correspond to the illuminance $\left(\mathrm{lm} \mathrm{cm}^{-2}\right)$. The control panel of the Ulbricht Sphere just indicates the detected lumen $(\mathrm{lm})$ by the light sensor and $\mathrm{AC}$ power consumption $\mathrm{W}_{\text {tube }}$ of the $\mathrm{AC}$ driving circuit. The $\mathrm{W}$ of the control panel does not relate with the generation energy of the lights in the FL tubes. The definitions of the $\mathrm{W}_{\text {tube }}$ and $\mathrm{W}$ will be in late in this report. The wrong determinations on the control panel of the Ulbricht Sphere deliberately give the luminous efficiency $\left(1 \mathrm{~m} \mathrm{~W}^{-1}\right)$, instead of the illuminance $\left(\mathrm{lm} \mathrm{m}^{-2}\right)$. The present Ulbricht Sphere has the totally wrong arrangement in the study on the light source and the evaluation of the performance of the FL tubes. Accordingly, the determined luminous efficiency ( $\mathrm{lm} \mathrm{W}$ ) from the control panel of the Ulbricht Sphere gives the invalided information for the evaluation of the practical FL tubes. The FL tubes essentially hold the unrivaled advantages over other solid light sources as the illumination source. The invalided results by the improperly modified Ulbricht Sphere conceal the unrivaled advantages of the FL tubes. We will reveal the concealed advantage of the lighted FL tubes.

The capability of the light sources should be determined by the illuminance $\left(1 \mathrm{~m} \mathrm{~m}^{-2}\right)$ or luminance $\left(\mathrm{cd} \mathrm{m}^{-2}\right)$. It is impossible to find out the quantitative calculation of the illuminance $\left(\mathrm{lm} \mathrm{m}^{-2}\right)$ from the determined luminance $(\mathrm{lm})$ in the improperly modified Ulbricht Sphere shown in Figure 3. We must find out the illuminance $\left(1 \mathrm{~m} \mathrm{~m} \mathrm{~m}^{-2}\right)$ from the information on the control panel of the modified Ulbricht Sphere for the evaluation of the FL tubes.

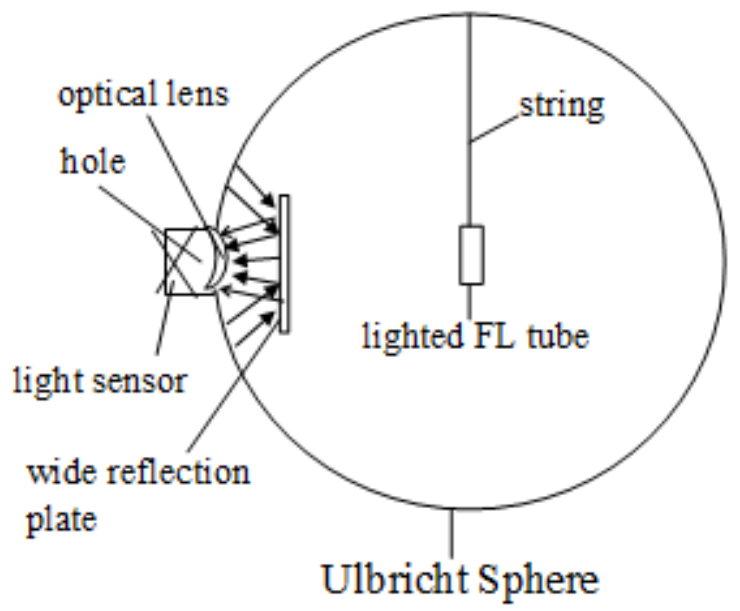

Figure 3. Illustration of incorrect arrangement of a large reflection plate that sets in front of hole of light sensor with the collection lens.

It has found a collection factor from the detected light intensity of the modified Ulbricht Sphere in Figure 3, after many trials and errors. The detected lumen in the Figure 3 enhances to $1.6 \times 10^{3}$ times of the light intensity on the unit surface area $\left(\mathrm{cm}^{2}\right)$ of the inner wall of the Ulbricht Sphere. The real illuminance $\left(1 \mathrm{~m} \mathrm{~cm}{ }^{-2}\right)$ is $6 \times 10^{-4} \mathrm{~cm}^{-2}$ times of the detected lumen $(\mathrm{lm})$ of the control panel. The enhancement changes with the individuals of the modified Ulbricht Sphere. We may calculate the real illuminance $\left(1 \mathrm{~m} \mathrm{~m}^{-2}\right)$ of the FL tube by the multiplication of $6 \mathrm{~m}^{-2}\left\{=1 \times 10^{4} \times\left(1.6 \times 10^{3} \mathrm{~m}^{2}\right)^{-1}\right\}$ to the detected lumen on the control panel of the studied Ulbricht Sphere. For instance, if you have the $500 \mathrm{~lm}$ on the control panel, the illuminance of the FL tube is $3000 \mathrm{~lm} \mathrm{~m}^{-2}$ (=6 500 $1 \mathrm{~m} \mathrm{~m}^{-2}$ ). Now we may quantitatively evaluate the performance of the FL tubes as the illuminance $\left(1 \mathrm{~m} \mathrm{~m}^{-2}\right)$ by the use of the modified Ulbricht Sphere.

\section{Moving Electron Multiply Excites Floating Hg Atoms in FL Tubes}

The light intensities from the lighted FL tubes originate from the UV lights from the excited $\mathrm{Hg}$ atoms in the positive column by the moving electrons. The volume of the Ar gas and the amount of the moving electrons in the positive column are the constant in the given type of the lighted FL tubes. The variable factor is the evaporated $\mathrm{Hg}$ atoms from the droplets on the phosphor screen. The ionization of the Ar atoms in the positive column by the moving electrons is solely the heat source by the change in the entropy. The temperatures of the positive column determine the amount of the evaporated $\mathrm{Hg}$ atoms in the positive column from the $\mathrm{Hg}$ droplets on the phosphor screen. The details are the below:

The FL tubes are assembled with five parts: (i) vacuum-sealed glass tube, (ii) $\mathrm{Ar}$ gas pressures, (iii) $\mathrm{Hg}$ droplets in a few mg, (iv) a pair of the electrodes and (v) phosphor screen. The experimental FL tubes were made with the arrangement of the components listed above. The acceptable FL tubes had been optimized empirically, not theoretically. The analysis of the experimental results of the FL tubes had made with the analogues with the solid-state science.

The Ar gas space in FL tubes quite differs from solids. The $\mathrm{Ar}$ atoms in FL tube float in vacuum with the separation distance of $2 \times 10^{-7} \mathrm{~m}$ with the Maxwell-Boltzmann distribution. The electrons move on in the superconductive vacuum space between $\mathrm{Ar}$ atoms without the thermal perturbation from the neighboring Ar atoms. The moving electrons in the lighted FL tubes do not have the Joule Heat. The study on the FL tubes belongs to the vacuum physics, rather than solid-state physics. On the other hand, the vacuum space of the solids is determined by the lattice sites that is around $10^{-10} \mathrm{~m}$. The moving electrons in solids inevitably have the electric resistance caused by the thermal perturbation from the thermally vibrating atoms at the lattice sites. The thermal perturbation generates the electric resistance for the moving electrons. The moving electrons in the solids unavoidably have the Joule Heat that is given by $\mathrm{I}^{2} \mathrm{R}$ where $\mathrm{I}$ is flowing electrons (electric current) and $\mathrm{R}$ is the electric resistance.

The large difference between FL tube and lighting solids is the generation of the lights. The lights in the solids generate 
with the recombination of the injected electron and hole at the luminescent centers in the solids. The maximum quantum efficiency $\eta_{\mathrm{q}}$ of the moving electron in the solid is 1.0. But this is impossible in the solids. The moving electrons in the lighting solids inevitably lost the energy by the Joule Heat. Consequently the $\eta_{\mathrm{q}}$ of the solid lighting devices is lower than 1.0. In many cases, the $\eta_{\mathrm{q}}$ is around $0.5 ; \eta_{\mathrm{q}} \approx 0.5$.

The lights in the FL tubes originate from the excitation of the floating $\mathrm{Hg}$ atoms in vacuum by the moving electrons. The lights are not generated by the recombinations of the injected electrons and holes at the luminescence centers. As the moving electron in the superconductive vacuum meets the floating $\mathrm{Hg}$ atom, the moving electron cannot penetrate into the $\mathrm{Hg}$ atom. The moving electron receives the strong Coulomb's repulsion from the orbital electrons of the $\mathrm{Hg}$ atom. The moving electron that has the Coulomb's repulsion gives some amount of the kinetic energy to the $\mathrm{Hg}$ atoms. The electrons in the electron shells of the $\mathrm{Hg}$ atoms rise to the higher energy levels in the $\mathrm{Hg}$ atoms. This is the excitation mechanisms of the floating $\mathrm{Hg}$ atoms in the vacuum. The excited $\mathrm{Hg}$ atom generates the UV lights by the electron transitions from the excited energy levels $\left({ }^{6} p_{1}\right.$ and $\left.{ }^{1} p_{1}\right)$ to the grand state ${ }^{6} \mathrm{~S}_{0}$ of the $\mathrm{Hg}$ atom. This is the excitation mechanism of the $\mathrm{Hg}$ atoms in the Ar gas space.

The repulsed electron from the Ar atom does not disappear in the Ar gas space. The repulsed electron stays in the superconductive vacuum. The repulsed electron from the $F_{\text {orb }}$ of the Ar atom is the scattered electron from the longitudinal direction. As the scattered electron in the vacuum is under the $F_{\text {vect }} \geq F_{\text {orb }}$, the electron is under the $F_{\text {vect }}$. The electron moves again the longitudinal direction. The electron under the $F_{\text {vect }}$ gains the kinetic energy from the $F_{\text {vect }}$. The accelerated electron has a chance to meet other $\mathrm{Hg}$ atom. By the repetition of the Coulomb's repulsion and recovering of the kinetic energy under the $F_{\text {vect }}$ in the vacuum, the electrons from the cathode never disappear in the positive column up to the anode. Consequently, the moving electron has the multiple excitations of the $\mathrm{Hg}$ atoms in the Ar gas space in the positive column. The number of the excitations of the $\mathrm{Hg}$ atoms by one moving electron in the positive column of the FL tube is calculable with the moving model described above. The calculated number per unit $\mathrm{Ar}$ gas space $\left(\mathrm{m}^{-3}\right)$ gives the astronomical quantum efficiency $\eta_{\mathrm{q}}=10^{12}$ photons $\left(\mathrm{m}^{3}, \mathrm{~s}\right)^{-1}$ in the commercial FL tube [4]. The DC electric current in the internal DC electric circuit is $2 \times 10^{-4} \mathrm{~A}$ maximum that contains $1 \times 10^{15}$ electrons per second $\left\{=2 \times 10^{-4} \mathrm{~A} \times(1.6 \mathrm{x}\right.$ $\left.\left.10^{-19} \mathrm{~s}\right)^{-1}\right\}$. By the considering the volume of the FL tube, we may calculate the number of the emitted photons per the 40W-HCFL tube per second. The 40W-HCFL tube (T-10) emits the $10^{25}$ photons per second $\left(=10^{15} \times 10^{12} \mathrm{x} \mathrm{V}_{\text {posi }} \mathrm{X} \mathrm{s}^{-1}\right)$. The calculated $10^{25}$ photons per second and per FL tube accord with the emitted photons of the practical 40W-HCFL tubes [4] As described above, the moving electrons in the Ar gas space in the lighted FL tubes and the generation mechanism of the lights quite differ from the moving electrons in the solids. The astronomical quantum efficiency $\left\{\eta_{\mathrm{q}}=10^{12}\right.$ photons $\left.\left(\mathrm{m}^{3}, \mathrm{~s}\right)^{-1}\right\}$ is the unrivaled advantage of the FL tubes. The unrivaled advantage of the FL tube has concealed by the evaluation by the invalided luminous efficiency $\left(\mathrm{lm} \mathrm{W}^{-1}\right)$ and the active $\mathrm{AC}$ power consumption $\left(\mathrm{W}_{\text {act }}\right)$ of the external $\mathrm{AC}$ driving circuit. The $\mathrm{W}_{\text {act }}$ does not relate with the generation of the lights in the FL tubes.

\section{Diameter of Positive Column Determined by $\mathbf{F}_{\text {phos }}$}

The moving electrons in the Ar gas space in the positive column are influenced with the localized electric field. Before the quantitative determination of the lighted $\mathrm{Hg}$ atoms in the positive column, it is a better way to clarify the formation mechanisms of the positive column and the defined diameter of the positive column in the Ar gas space of the lighted FL tubes.

The volume of the positive column by the given FL tubes changes with the localized electric field from the phosphor screen, $\mathrm{F}_{\text {phos. }}$. In the past, the phosphor screens in the FL tubes have just considered as the transducer from the UV lights to the lights in the visible spectral wavelengths. The quality of the phosphor screen as the transducer has been optimized under the UV lights from the short UV lamp. The electric charges on the phosphor particles in the vacuum have disregarded in the study on the FL tubes. The strongly restricting factor of the performance of the lighted FL tube is the localized $F_{\text {phos }}$ of the phosphor screen, [5, 6, and 7]. The $F_{\text {phos }}$ has the vertical electric field against to the longitudinal $\mathrm{F}_{\text {vect }}$. The $\mathrm{F}_{\text {phos }}$ determines the volume in which the electrons exclusively move on in the Ar gas space. The determined volume in the Ar gas space is called as the positive column. Naturally the lighted HCFL tubes have the gap between the positive column and phosphor screen. The depths of the gap determine followings;

(a) Outer diameters of the practical FL tubes,

(b) Temperature of the positive column,

(c) Optical absorption of the UV lights before reaching to the phosphor screen, and

(d) Ar gas pressures in the practical FL tubes.

As the moving electrons are reaching to the phosphor screen, the moving electrons receive the Coulomb's repulsion from $\mathrm{F}_{\text {phos }}$. The moving electrons in the Ar gas space never reach to the phosphor screen. The conductivity of the phosphor screen does not involve in the moving electrons in the Ar gas space. The repulsed electrons by the $F_{\text {phos }}$ move on in the volume of the Ar gas space that is defined by $F_{\text {vect }} \geq F_{\text {phos }}$. Followings are an example of the defined diameter of the positive column in the lighted FL tube.

The $\mathrm{Ca}_{3}\left(\mathrm{PO}_{4}\right)_{4}(\mathrm{~F}, \mathrm{Cl}): \mathrm{Sb}: \mathrm{Mn}$ white emitting phosphor screen was the typical phosphor screen of the FL tube before 1975. The $\mathrm{Ca}_{3}\left(\mathrm{PO}_{4}\right)_{4}(\mathrm{~F}, \mathrm{Cl}): \mathrm{Sb}: \mathrm{Mn}$ phosphor screens only emit the brilliant photoluminescence (PL). The phosphor screen cannot use in the cathodoluminescence (CL) in the vacuum devices such as CRT with the darkness. The PL phosphor screens are the electric insulator that contains many trapped electrons in the activators (e.g., $\mathrm{Sb}$ and $\mathrm{Mn}$ ) in the 
phosphor particles. The trapped electrons generate the $\mathrm{F}_{\text {phos. }}$ In the case of the $\mathrm{Ca}_{3}\left(\mathrm{PO}_{4}\right)_{4}(\mathrm{~F}, \mathrm{Cl}): \mathrm{Sb}: \mathrm{Mn}$ phosphor screens, the depth of the gaps is deeper than $4 \times 10^{-3} \mathrm{~m}$. The depth of the gap determines the outer diameter of the practical HCFL tubes wider than $3.2 \times 10^{-2} \mathrm{~m}$ (T-10). We must know the reason of the optimized diameter (T-10) of the practical $40 \mathrm{~W}-\mathrm{HCFL}$ tubes.

We may calculate the diameter of the positive column in the 40W-HCFL tube. The thickness of glass tube is $1 \times 10^{-3} \mathrm{~m}$. The inner diameter of T-10 glass tube is $3.0 \times 10^{-2} \mathrm{~m}$. The total depth of the gap in the inner FL tubes is deeper than $8 \times 10^{-3} \mathrm{~m}$ $\left(=4 \times 2 \times 10^{-3} \mathrm{~m}\right)$. The inner diameter does not correspond to the diameter of the positive column. The calculated diameter of the positive column is narrower than $2.2 \times 10^{-2} \mathrm{~m}\{=(3.0-$ $\left.0.8) \times 10^{-2} \mathrm{~m}\right\}$ in the HCFL tubes (T-10). As the FL tube has the length of $1.0 \mathrm{~m}$, the inner volume of the glass tube, $\mathrm{V}_{\text {glass }}$, is $7.0 \times 10^{-4} \mathrm{~m}^{3}\left(=2 \pi \mathrm{r}^{2} \times 1.0\right)$. The volume of the positive column $\mathrm{V}_{\text {posi }}$ is $3.8 \times 10^{-4} \mathrm{~m}^{2}$. The ratio of the $\mathrm{V}_{\text {posi }} \times\left(\mathrm{V}_{\text {glass }}\right)^{-1}$ is $0.54\left(=3.8 \times 7.0^{-1}\right)$. The electrons move on in the Ar gas space is a nearly half of the total Ar gas in the 40W-HCFL tube. The lights in the 40W-HCFL tube are generated in the half volume of the Ar gas space that is determined by the Ar gas pressures. The gap contains the left Ar gas that is 0.46 of the $\mathrm{V}_{\text {glass }}$.

\section{Temperatures of Positive Column Determine Illuminance of FL Tubes}

The positive column in the lighted FL tubes is surrounded with the good thermal insulator of the Ar gas at $900 \mathrm{~Pa}(\sim 7$ Torr). Thermal conductivity of Ar gas is $4 \times 10^{-7} \mathrm{cal}(\mathrm{cm}, \mathrm{s}$, $\mathrm{T})^{-1}$. The origin of the light source of the lighted FL tubes is the UV lights; $254 \mathrm{~nm}$ and $183 \mathrm{~nm}$, from the excited $\mathrm{Hg}$ atoms. The $\mathrm{Hg}$ atoms in the vacuum-sealed FL tubes are the $\mathrm{Hg}$ droplets on the phosphor screen. $\mathrm{The} \mathrm{Hg}$ atoms evaporate from the $\mathrm{Hg}$ droplets on the phosphor screen to the positive column. The heat source in the lighted FL tubes is assigned as the ionization of the Ar atoms by the change in the entropy. The Hg droplets on the phosphor screen should heat up by the heating from the positive column. The temperatures of the positive column mainly determine the amount of the generated lights. The high temperatures of the positive column generate the more UV lights. Here is a problem that the Ar gas in the gap contains the unexcited $\mathrm{Hg}$ atoms. The unexcited $\mathrm{Hg}$ atoms in the gap optically and efficiently absorb the UV lights emitted from the positive column before reaching to the phosphor screen. The absorbed UV lights by the gap are a half of the emitted UV lights in the positive column. The narrow diameter of the positive column and optical absorption of the $\mathrm{UV}$ lights by the unexcited $\mathrm{Hg}$ atoms in the gap determine the outer diameter of the lighted FL tubes as $3.2 \times 10^{-2} \mathrm{~m}$ (T-10. The HCFL tubes with the $\mathrm{Ca}_{3}\left(\mathrm{PO}_{4}\right)_{4}(\mathrm{~F}, \mathrm{Cl}): \mathrm{Sb}: \mathrm{Mn}$ phosphor screens are operated with the conditions described above. The calculation results inform us that if the gap is shallower than 5 $\mathrm{x} 10^{-4} \mathrm{~m}$, the diameter of the positive column extends to the near inner diameter of the FL tube. Consequently, the optical absorption of the unexcited $\mathrm{Hg}$ atoms in the gap becomes to the negligible level. Then the brighter FL tubes may produce with the diameter narrower than $1 \times 10^{-2} \mathrm{~m}$.

After 1975, the phosphor screens are made with the blend mixture of the tri-color rare earth phosphor powders that give the depths of the gap of $3 \times 10^{-4} \mathrm{~m}$. The shallow gap allows the outer diameters of the FL tubes narrower than the $9.5 \times 10^{-3} \mathrm{~m}$ (T-3), depending on the quality of the phosphor screens, especially red phosphor particles. If the depth of the gap is the $3 \times 10^{-4} \mathrm{~m}$, the build-up curve of the light intensities will saturate within a few minutes. If the phosphor powders contain the solid binder in the low melting temperatures, the $\mathrm{F}_{\text {phos }}$ sensitively change with the amount of the solid binders and kinds of the solid binders. The solid binders are poisons for the depths of the gap. Many commercialized compact-HCFL tubes have the outer diameter in $9.5 \times 10^{-3} \mathrm{~m}$ (T-3) that indicates the small value of the $\mathrm{F}_{\text {phos. }}$.

$\mathrm{The} \mathrm{Hg}$ atoms float in the vacuum in the Ar gas space with the ratio of $10^{-3} \mathrm{Hg}$ atom per one Ar atom. The ratio changes with the temperatures of the Ar gas space in the positive column; high concentrations of the evaporated $\mathrm{Hg}$ atoms in the high temperatures of the Ar gas space. The temperatures of the Ar gas space in the positive column determine the amount of the evaporated $\mathrm{Hg}$ atoms from the $\mathrm{Hg}$ droplets on the phosphor screen. Each evaporated $\mathrm{Hg}$ atom in the positive column generates lights in the lighted FL tubes. The determination of the temperatures of the positive column may give a figure of the merit of the lighted FL tubes.

The inner wall of the FL tubes is covered with the opaque phosphor screen. There is no way to set the thermal detector in the positive column in the lighted FL tubes. It has found that the temperature of the positive column may detect by the infrared thermometer from the outside of the FL tubes. We have determined the temperatures of the positive column by the infrared thermometer. The temperatures of the positive column of many commercial compact-HCFL tubes are around $35^{\circ} \mathrm{C}$ to $40^{\circ} \mathrm{C}$. We have selected the compact-HCFL tubes with the temperatures of the positive column above $60^{\circ} \mathrm{C}$. The selected compact-HCFL tubes in the Ulbricht Sphere have 3 to 5 minutes for the saturation of the build-up curve of the light intensities from the phosphor screen, indicating the gap narrower than $5 \times 10^{-4} \mathrm{~m}$.

\section{External AC Driving Circuit of FL Tubes Closes with Induced AC Current}

Before the discussion of the illuminance $\left(\mathrm{lm} \mathrm{m}^{-2}\right)$, we must clarify the active power consumption, the $\mathrm{W}_{\text {act }}$, of the $\mathrm{AC}$ driving circuit of the lighted FL tubes.

It has obstinately believed for a long time that the external AC driving circuit in the lighted FL tubes is electrically closed by the electron flow in the Ar gas space in the lighted FL tubes. The cathode electrode of the heated $\mathrm{BaO}$ particles directly injects the electrons to the $\mathrm{Ar}$ gas space and the anode electrode of the $\mathrm{BaO}$ particles collects arrived electrons from the Ar gas space. Beside the electron flow, the moving electrons in the Ar gas space are modified by the AC electric 
field from the electrodes $[2,3]$.

The injection of the thermoelectrons from the heated $\mathrm{BaO}$ particles to the Ar gas space is a hypothesis. The hypotheses had supported by the current waveform of the oscilloscope, which detected at the electrodes of the lighted FL tubes. The oscilloscope does not have the current sensor. The oscilloscope has only voltage sensor. The waveform of the screen of the oscilloscope is actually the change in the electric potential at the electrodes of the capacitor $C_{\text {tube. }}$. The hypothesis is not proved by the scientific evidence. The scientific evidences are below:

Recently, it has revealed that the disparities of the external AC driving circuit and internal DC electric circuit coexist in the operation of the lighted FL tubes as unit $[8,9]$. There is no electron flow between two circuits. Two electric circuits conjugate with the electric field from the electrodes of the external AC electric circuit. The external AC driving circuit is electrically closed by the induced $\mathrm{AC}$ current from the capacitor $\mathrm{C}_{\text {tube }}$. The power consumption $\mathrm{W}_{\text {act }}$ of the $\mathrm{AC}$ driving circuit is not related with the generation energy of the lights from the FL tubes. The lights are solely generated by the excitation of the $\mathrm{Hg}$ atoms by the moving electrons from the cathode to the anode of the internal DC electric power generator [9]. The power consumption of the internal DC electric circuit, $\mathrm{W}_{\mathrm{DC}}$, is less than $0.02 \mathrm{~W}(=100 \mathrm{~V} \times 0.2 \mathrm{~mA})$ that is a negligibly small. The AC electric power consumption, $\mathrm{W}_{\text {act }}$, of the commercial HCFL tubes are higher than $15 \mathrm{~W}$, depending on the (a) diameters of the glass tubes, (b) lengths of HCFL tubes, and (c) Ar gas pressures.

The external AC driving circuit is closed by the AC induced current $(\approx 0.3 \mathrm{~A})$ from the capacitor $\mathrm{C}_{\text {tube }}$, which is formed by floating $\mathrm{Ar}^{1+}$ in vacuum. The induced AC current is not by the electron flow in the Ar gas space of the FL tubes. The external AC driving circuit is electrically closed with the induced electric current that corresponds to the displacement of the distribution of the electrons in the inside of the electron shell of $\mathrm{Ar}^{1+}$. The $\mathrm{C}_{\text {tube }}$ is formed with the ionized Ar atoms $\left(\mathrm{Ar}^{1+}\right)$. Naturally, the determined $\mathrm{W}_{\text {act }}$ does not relate with the energy for the generation of the lights in the FL tubes. The $\mathrm{W}_{\text {act }}$ is inclusively determined by the capacitance of the $\mathrm{C}_{\text {tube }}$. The reduction of the $\mathrm{W}_{\text {act }}$ is other important subject. We must concentrate here the illuminance $\left(1 \mathrm{~m} \mathrm{~m} \mathrm{~m}^{-2}\right)$ of the compact-HCFL tubes.

\section{Determination of Illuminance of Commercial Compact-HCFL Tubes}

Recently it has found that some of the commercial compact-20W-HCFL tubes on the lighting stores in China have the temperatures of the positive column at around $60 \pm$ $10^{\circ} \mathrm{C}$. The high temperatures of the positive column are only achieved by the high Ar gas pressures. The estimated Ar gas pressure is $2 \times 10^{3} \mathrm{~Pa}$ (=15 Torr). Another clue for the selection of the commercial compact-20W-HCFL tube is the depth of the gap. The depth of the gap can be estimated from the build-up curve of the lumen ( $1 \mathrm{~m})$ in the Ulbricht Sphere. The build-up curves of the lumen $(\mathrm{lm})$ of the selected compact-HCFL tubes from the stores saturate within 5 minutes, indicating the shallow gap $\left(5 \times 10^{-4} \mathrm{~m}\right)$. The build-up curve of the lumen in the Ulbricht Sphere slightly goes down after the saturation. The slightly going down of the lumen with the running time is cooling of the outer glass wall of the lighted FL tubes by the convection of the cold air (room temperature) in the large volume of the Ulbricht Sphere. The slightly decreased curve again increases to the initial saturation level after the heat up the air in the Ulbricht Sphere. We may take the lumen $(1 \mathrm{~m})$ at the initial saturated curve.

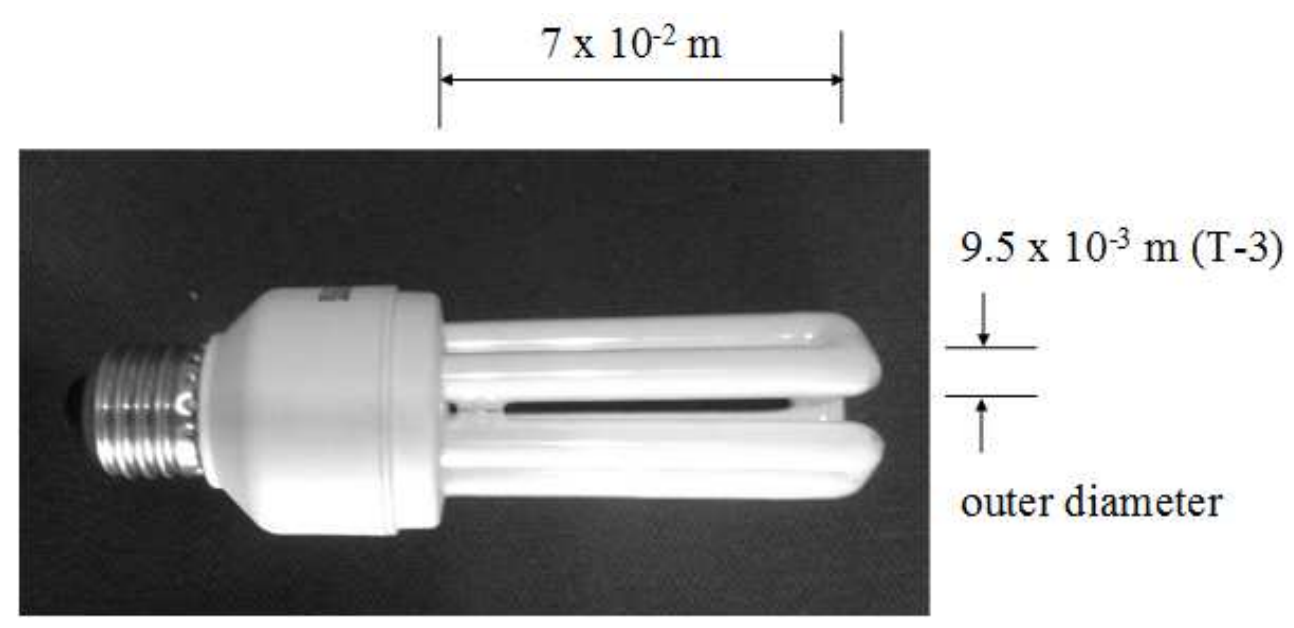

Figure 4. Photograph and dimension of $20 \mathrm{~W}$ compact-FL tube.

Figure 4 shows the photograph and dimensions of the tested 20W-HCFL tube. The outer diameter of the FL tube is $9.5 \mathrm{x}$ $10^{-3} \mathrm{~m}(\mathrm{~T}-3)$. The thickness of the glass tube is $0.5 \times 10^{-3} \mathrm{~m}$. The inner diameter of the glass tube is $8.5 \times 10^{-3}$, and total length 1 of the FL tubes is $2.8 \times 10^{-1} \mathrm{~m}\left(=4 \times 7 \times 10^{-2} \mathrm{~m}\right)$. The inner volume of the tested compact-HCFL tube $\left(\mathrm{V}_{\text {comp }}\right)$ is $1.6 \mathrm{x}$
$10^{-5} \mathrm{~m}^{3}\left\{=\pi \mathrm{r}^{2} \times 1=\pi \times\left(8.5 \times 10^{-3}\right)^{2} \times 2.8 \times 10^{-1} \times 4^{-1}\right\}$. We cannot figure out the exact $\mathrm{Ar}$ gas pressures of the tested compact-20W-HCFL tube. The systematic study on the Ar gas pressures, heated temperatures of $\mathrm{Ar}$ gas space and $\mathrm{W}_{\text {act }}$ remains for a future study.

We have selected the compact-20W-HCFL tubes from the 
lighting stores with the temperatures of the positive column higher than $65^{\circ} \mathrm{C}$ by the infrared thermometer. By the use of the selected compact-20W-HCFL tubes, we have determined (a) the lumens $(\mathrm{lm})$ of the control panel of the tested Ulbricht Sphere, (b) illuminance $\left(\mathrm{lm} \mathrm{m}^{-2}\right)$, (c) operation temperature of the positive column in the lighted FL tubes after the saturation of the light intensity, and (d) AC power consumption, $\mathrm{W}_{\text {act }}$ of the external AC driving circuit.
The lumens $(\mathrm{lm})$ of the tested five compact HCFL tubes in the Ulbricht Sphere were $950 \pm 50 \mathrm{~lm}$ on the control panel. The $950 \mathrm{~lm}$ is not the illuminance $\left(\mathrm{lm} \mathrm{m}^{-2}\right)$. The determined illuminance $\left(\mathrm{lm} \mathrm{m}^{-2}\right)$ of the $20 \mathrm{~W}$-compact-FL tubes is $5700 \pm$ $300 \mathrm{~lm} \mathrm{~m}^{-2}\left(=950 \mathrm{~lm} \mathrm{x} 6 \mathrm{~m}^{-2}\right)$. The $\mathrm{W}_{\text {act }}(=20 \mathrm{~W})$ of the compact-HCFL tubes have determined at input terminals. Table 1 summarizes the determined results.

Table 1. Properties of commercial 20-W compact-FL tubes.

\begin{tabular}{|c|c|c|c|}
\hline detected lumens & Illuminance $\left(\mathrm{Im} \mathrm{\textrm {m } ^ { - 2 } )}\right.$ & operation $\mathrm{T}\left({ }^{\circ} \mathrm{C}\right)$ & $\mathbf{W}_{\text {act }}(\mathrm{W})$ \\
\hline $950 \pm 50(\mathrm{~lm})$ & $5700 \pm 300$ & \pm 10 & $20 \pm 1$ \\
\hline
\end{tabular}

We have measured the temperatures of the positive column of many commercial compact HCFL tubes in the stores on the world. The temperatures of many compact-HCFL tubes in the same size were around $35 \pm 5^{\circ} \mathrm{C}$ and the $\mathrm{W}_{\text {act }}$ is below $15 \mathrm{~W}$. Naturally, they have the illuminance below $3000\left(1 \mathrm{~m} \mathrm{~m}^{-2}\right)$, depending on the producers. The low illuminance and $\mathrm{W}_{\text {act }}$ are caused by the low Ar gas pressure in the FL tubes. We do not take those compact-FL tubes in this study with the reason that the FL producers do not have the chance to increase the illuminance and the short operation life less than 500 hours.

\section{Illumination of Given Room by Examined Compact-HCFL Tubes}

The human eyes adjust for the daytime scenery under the slightly overcastting sky for 5 million years. The daytime scenery is made by the scattered lights of the thin cloud. The daytime scenery is not made by the direct light from the sun. The scattered lights in the large size of the lighting source are preferable for the illumination purpose of the rooms with the

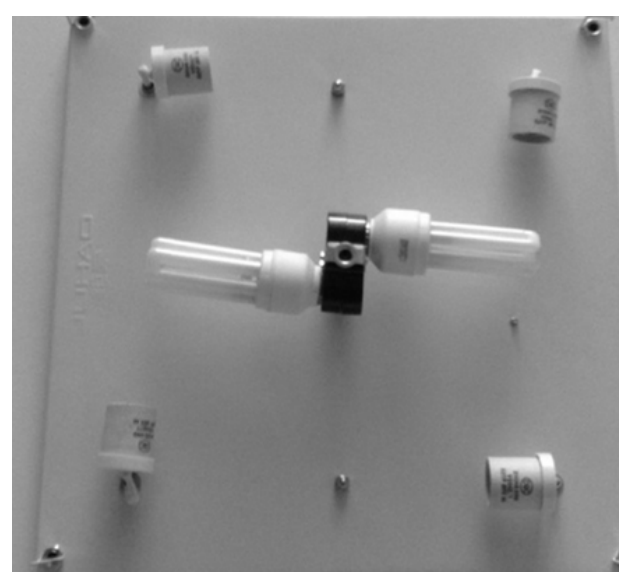

(A) less shadow. The experiments of the illumination of the rooms are made with the widely scattered lights from the FL tubes, even though the phosphor screen emits the scattered lights. The furniture in a given room of the house or desktop of the office of the large buildings should be illuminated with the daytime scenery from the widely scattered light source for the comfortable observation by the eyes. At present time, users of many compact-FL tubes directly light up with the compact-HCFL tubes. The compact-FL tubes should light up with the opaque plastic cover in the large size for the generation of the scattered lights, like as the clouds in the sky. The comfortable illuminance level by the scattered lights is $330\left(\mathrm{~lm} \mathrm{~m}^{-2}\right)$ with the less shadow. The single compact $20 \mathrm{~W}-\mathrm{HCFL}$ tube has the illuminance of $5700\left(\mathrm{~lm} \mathrm{~m}^{-2}\right)$ that has the capability of the illumination of the $17 \mathrm{~m}^{2}$ room $(=5700 \mathrm{x}$ $\left.330^{-1}\right)$. The two compact-20W-HCFL tubes in the opaque plastic cover may illuminate the furniture in the $30 \mathrm{~m}^{2}$ room in the house under the illuminance $\left(380 \mathrm{~lm} \mathrm{~m}^{-2}\right)$. Followings are the experimental proof of the usage of the compact-FL tubes as the illumination source.

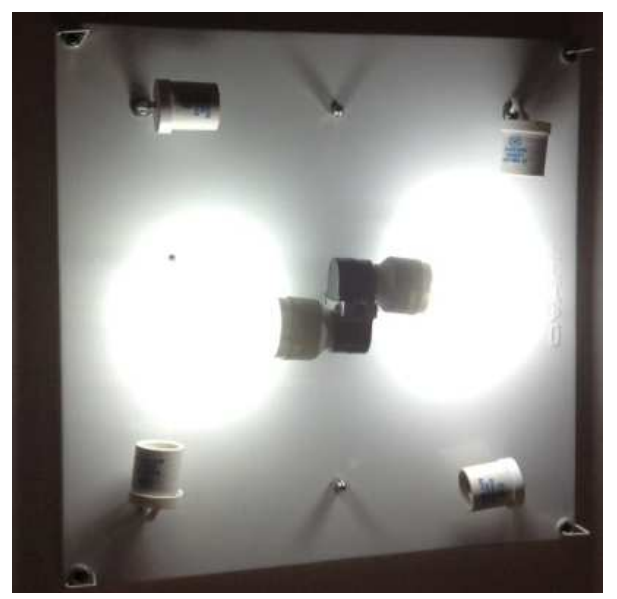

(B)

Figure 5. Photographs of installation of two compact-FL tubes on lighting sockets on ceiling. (A) is unlighted compact-FL tubes, and (B) is lighted FL tubes.

Figure 5 shows photographs of the installation of two 20W-compact-FL tubes on the ceiling of the $30 \mathrm{~m}^{2}$ living room of the house. Figure 5-A is photograph of the unlighted compact-FL tubes. Figure 5-B is photograph of the lighted compact-FL tubes without the opaque plastic cover. The lighted compact-FL tubes are covered with the volume of the brighter lights from the FL tubes. We cannot see the lighted FL tubes. This is by the saturation of the sensitivity of the camera sensor and the observation by the eyes. The furniture is not uniformly illuminated by the direct lights from the FL tubes in Figure 5-B. The FL tubes light up very large spots that do not large enough to give the uniform scenery under the slightly 
overcastting sky.

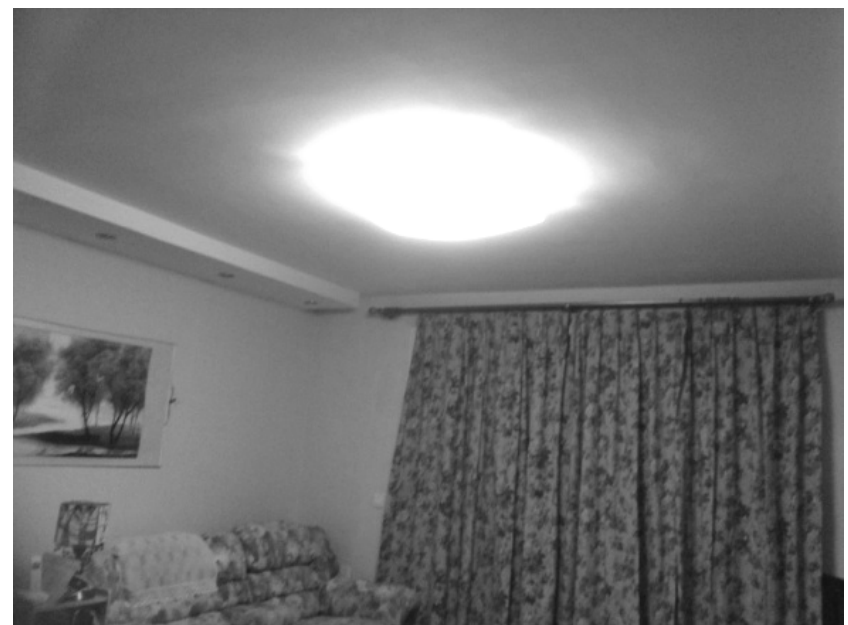

(A)

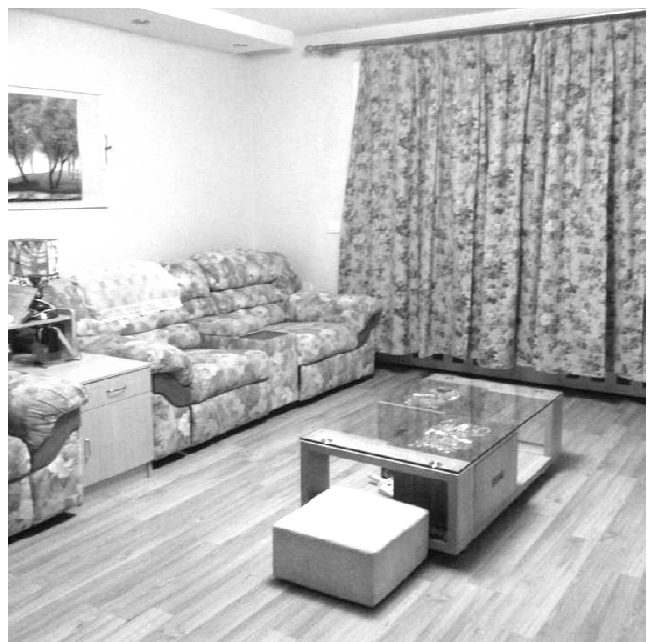

(B)

Figure 6. Photographs of lighted FL tubes in opaque cover for scattering of lights. (A) Photograph that the camera focuses on scattered light source on ceiling. (B) Photograph is the illuminated room with scattered lights from the light source.

Figure 6 shows photographs of the lighting source that is covered with a white and opaque plastic cover in a large size $0.36 \mathrm{~m}^{2}\left(=0.6 \times 0.6 \mathrm{~m}^{2}\right)$. The light source extends to $0.6 \times 0.6$ $\mathrm{m}^{2}$ from the size of the compact-FL tubes. Figure $6(\mathrm{~A})$ is the photograph that the camera is automatically focused on the lighting source on the ceiling of the room. The lights from the compact-HCFL tubes widely and uniformly scatter on the opaque plastic cover. The scattered lights by the opaque cover become a wide light source. The camera automatically adjusted to the light intensity of the scattered light source that is the saturation range of the sensitivity of the camera. The view of the camera should takes out the lighting source. Figure 6 (B) is the photograph that shows the furniture in the illuminated room without the focus of the camera to the light source. The photograph in Figure 6 (B) shows the comfortable scene of the furniture that is illuminated with the illuminance (380 $\mathrm{lm} \mathrm{m} \mathrm{m}^{-2}$ ). The room looks like the uniform illuminance with less shadow. This is the daytime scenery under the slightly overcastting sky. The illumination level has confirmed by the open of the window curtain at the noon time. The illuminance level of the room by the 2 compact-HCFL tubes is slightly brighter than the illuminance of the outside scenery through the window facing on the north sky. The furniture in the room smaller than $20 \mathrm{~m}^{2}$ may illuminate with a single compact-FL tube $\left(5700 \mathrm{~lm} \mathrm{~m}^{-2}\right)$ with the opaque large cover with Figure 6 (A), not shown in photograph. You may calculate the illuminance of the $20 \mathrm{~m}^{2}$ room with the $285 \mathrm{~lm}$ $\mathrm{m}^{-2}\left(=5700 \mathrm{~lm} \mathrm{~m}^{-2} \times 20^{-1}\right)$ that is acceptable in the practical activity in the room during the night time. For instance, your eyes may comfortably watch the TV screen in the illuminated room at night time.

Many compact-FL tubes at present time directly install in the many tiny holes on the ceiling. The lights from the compact-FL tubes in the hole illuminate the large spot on the floor. This is not gentle illumination for the eyes and it is not good way for the saving of the electric power consumption.
You may select the compact-FL tubes that the positive column has the temperatures above $60^{\circ} \mathrm{C}$. The selected compact-FL tubes install on the ceiling with the opaque white cover in the wide size, like as Figure 6 (A). You may have the uniformly illuminated floor and furniture with the comfortable illuminance level like as Figure 6 (B) with the less number of the compact-FL tubes; possibly one third to one fifth of the present number of the compact FL tubes in the holes on the ceiling. You may have the comfortably illuminated room of the house and small office with the less $\mathrm{W}_{\text {act }}$. This is a way for the illumination of the room with the energy saving of the $\mathrm{W}_{\text {act }}$. The tested compact $20 \mathrm{~W}-\mathrm{HCFL}$ tubes claim the operation life of 10,000 hours. Many compact HCFL tubes on the stores have the illuminance below $3000\left(1 \mathrm{~m} \mathrm{~m}^{-2}\right)$ with the operation life less than 500 hours.

\section{Further Improvement of Lighted FL Tubes}

The AC power consumption of the $20 \mathrm{~W}$ compact-HCFL tube shown in Figure 4 may reduce to $10 \mathrm{~W}$, if one compact-HCFL tube separates to two HCFL tubes. The capacitance of the $\mathrm{C}_{\text {tube }}$ of each FL tube becomes a half. The electrodes of the W-filament coils are shorted at the outside of the FL tubes. And then the separated two FL tubes connect in series. The total capacitance is one fourth of the capacitance of the present $20 \mathrm{~W}$ compact-HCFL tube. The induced AC current is down to one fourth. The applied voltage to the series connection of the FL tubes may require the double of the $20 \mathrm{~W}$ compact-HCFL tube. The total AC power consumption may be $10 \mathrm{~W}_{\text {act }}$. Figure 7 shows the circuit diagram of the series connection of two compact-HCFL tubes with the inverter. The out-put voltage of the inverter directly connects to the electrodes of the compact-HCFL tubes. The application voltages may be double voltage to the single compact HCFL 
tube. If the high voltage is made with transformer from the inverter, the power consumption will reduce more low level from the present $\mathrm{W}_{\text {act }}$. The insertion of the extra solid capacitors, inductance and resistance to the circuit between output of the inverter and the electrodes of the FL tubes are unnecessary.

The operation life of the commercial 20W-compact HCFL tubes is claimed as 10,000 hours. If the compact-HCFL tube converts to the coil-EEFL tube in the glass diameter (T-3) with a high Ar gas pressure at $9 \times 10^{3} \mathrm{~Pa}(=\sim 70$ Torr $)$, the $\mathrm{W}_{\text {coil }}$ goes down to $0.3 \mathrm{~W}_{\text {act }}$ and the operation life will prolong to the time longer than $10^{6}$ hours. The study on the coil-EEFL tubes is underway.

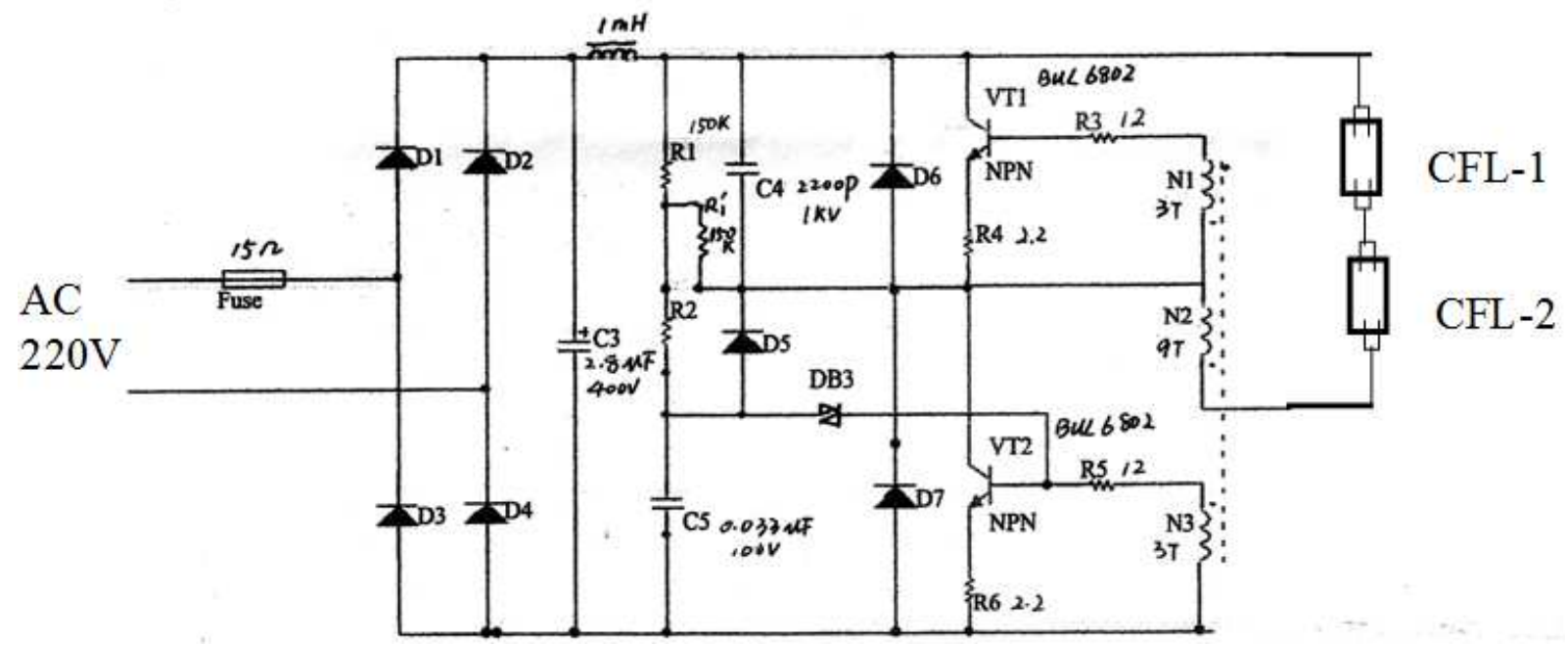

Figure 7. Circuit diagram of the series connection of two compact-HCFL tubes.

\section{Conclusion}

It has found that there are the commercial compact 20W-HCFL tubes, which have the advantage as the illumination source of the room in houses and desktop of offices in the large buildings. The selection criterion of the compact-20W-HCEF tubes is the temperatures of the positive column above $60^{\circ} \mathrm{C}$. The temperatures of the positive column can be determined by the infrared thermometer. The latent potential of the selected FL tubes should be evaluated by the illuminance $\left(1 \mathrm{~m} \mathrm{~m} \mathrm{~m}^{-2}\right)$ which is determined by the Ulbricht Sphere shown in Figure 1. It has found that the widely using Ulbricht Sphere in the study on and quality control of the FL tubes at the present time have intentionally modified with the wrong way in order to the evaluation of the FL tubes by the invalidated luminous efficiency $\left(1 \mathrm{~m} \mathrm{~W}^{-1}\right)$. After the correction of the Ulbricht Sphere, we had determined the high illuminance $5700\left(\mathrm{~lm} \mathrm{~m} \mathrm{~m}^{-2}\right)$ with the single compact $20 \mathrm{~W}-\mathrm{HCFL}$ tube. Then we may calculate the required number of the compact-HCFL tubes that illuminate the rooms in the houses and desktop in the offices with the illuminance $(330 \mathrm{~lm}$ $\mathrm{m}^{2}$ ) that human eyes adjust to the daytime activity for 5 million years. It has demonstrated that two compact 20W-HCFL tubes with the large opaque scattering cover illuminates the room in $30 \mathrm{~m}^{2}$ with the illuminance $(380 \mathrm{~lm}$ $\mathrm{m}^{2}$ ). The day-time scenery under the slightly overcastting sky is the illuminance $\left(330 \mathrm{~lm} \mathrm{~m}^{-2}\right)$.

The tested compact-HCFL tubes indicate the remaining a large room for the improvement by the advanced technologies of the FL tubes. By the corrected Ulbricht Sphere and by the removal of the invalided luminance efficiency $\left(\mathrm{lm} \mathrm{W}^{-1}\right)$, you may find the latent advantage of the FL tubes as the illumination source. The technologies of the present FL tubes are a premature technology. The exciting subjects of the FL tubes remain for a future study. They are (a) the increase in the illuminance $\left(\mathrm{lm} \mathrm{m}^{-2}\right)$ from FL tubes, (b) the reduction of the unnecessary $\mathrm{W}_{\text {act }}$ for the generation of the lights in FL tubes to nearly zero level, and (c) the operation life longer than $10^{6}$ hours. The improved FL tubes may have the unrivaled lighting source over the solid lighting sources.

\section{Acknowledgements}

The author withes to express his thank to Dr. Takao Toryu for his guidance to this project.

\section{References}

[1] F. Meyer, US Pat. 2,182,732 (1928).

[2] J. F. Waymouth, "Electric discharge lamps" MIT Press (1971).

[3] "Handbook of gas discharge", Japan. Inst. Elect. Engs., (1973).

[4] L. Ozawa and Y. Tian, "Calculation of the quantum efficiency of phosphor screen in CRTs and FL tubes", J. Inf. Dis., 11, pp 128-133, (2010).

[5] L. Ozawa, "Cathodoluminescence", Kodansha Science \& VCH, (1990).

[6] L. Ozawa "307 200 pixels $/ \mathrm{cm}^{2}$ resolution phosphor screen in monochrome CRT", Materials, Chemistry and Physics, 60, p 274-281 (1999). 
[7] L. Ozawa, "Cathodoluminescence \& Photoluminescence" CRC Press (2007).

[8] L. Ozawa and Y. Tian, "Coexistence of disparities of external AC driving circuit and internal DC electric circuit in operation of FL tube", J. China Ill. Engs, Soc., 6, p19-30 (2011)
[9] L. Ozawa, "Development of DC internal electric power generator in coil-FL tube", J. China Ill. Engs, Soc., 9, p86-93 (2014).

[10] L. Ozawa and Y. Tian, "A new 4G electron source for fluorescent lamp tubes", J. China Ill. Engs, Soc., 7, p58-65 (2012). 